\title{
APLICAÇÃO DO CUIDADO PALIATIVO NA ATENÇÃO PRIMÁRIA À SAÚDE: OBSTÁCULOS A SEREM VENCIDOS
}

\author{
APPLICATION OF PALLIATIVE CARE IN PRIMARY HEALTH CARE: \\ OBSTACLES TO BE OVERCOME
}

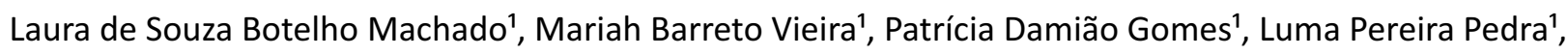
Myllena Cabral dos Santos ${ }^{1}$, Igor Leal Pena ${ }^{2}$

1 Acadêmicas de medicina da Faculdade de Medicina de Campos - FMC, Campos dos Goytacazes - RJ.

2 Professor auxiliar da cadeira de Medicina de Família e Comunidade, Faculdade de Medicina de Campos - FMC, Campos dos Goytacazes - RJ.

Seção de Revisão da Revista Científica da Faculdade de Medicina de Campos

Av. Alberto Torres, 217 - Centro, Campos dos Goytacazes - RJ, 28035-581

Endereço para correspondência: Laura de Souza Botelho Machado

Comendador José francisco sanguedo, 16 - ap 402, Campos dos Goytacazes - RJ

botelhomachado@gmail.com

Campos dos Goytacazes - RJ - 2020

\section{RESUMO}

O contato inicial da população com o Sistema Único de Saúde (SUS) ocorre principalmente na Atenção Primária à Saúde (APS). Que têm como princípios atenção a saúde física e mental integral do ser humano em sua individualidade e também no âmbito familiar e coletivo por meio dos cuidados essenciais. A atual conjuntura sociossanitária do Brasil mostra um momento de transição demográfica com aumento do envelhecimento da população e também mudanças epidemiológicas que registra aumento da prevalência de doenças crônico-degenerativas (enfermidades cardiovasculares e neoplasias) colocando em evidência os cuidados no final da vida, demandando a reorganização dos serviços de saúde. Nesse contexto, os cuidados paliativos (CP) podem ser desenvolvidos nos âmbitos ambulatoriais, de internação hospitalar e no domicílio do paciente, tais cuidados voltados para uma relação interpessoal de caráter multidisciplinar. Estas situações mostram o potencial tanto da APS e quanto da ESF (Estratégia de Saúde da Família). O único serviço de saúde que consegue estar ao lado dos pacientes e de sua

\begin{abstract}
The population's initial contact with the Unified Health System (SUS) occurs mainly in Primary Health Care (PHC). That has as principles attention to the integral physical and mental health of the human being in their individuality and also in the family and collective scope through essential care. The current socio-health situation in Brazil shows a moment of demographic transition with an increase in the aging of the population and also epidemiological changes that registers an increase in the prevalence of chronic-degenerative diseases (cardiovascular diseases and neoplasms), highlighting care at the end of life, demanding the reorganization of health services. In this context, palliative care $(P C)$ can be developed in the outpatient, hospitalization and patient's home settings, such care geared to a multidisciplinary interpersonal relationship. These situations show the potential of both PHC and FHS (Family Health Strategy). The only health service that manages to be with patients and their families to face the path of palliation with constant presence, guidance and welcome.
\end{abstract}


família para enfrentar o caminho da paliação com presença constante, orientação e acolhimento.

Palavras-chave: Cuidado Paliativo, Atenção primária e atenção básica

\section{INTRODUÇÃO}

A Atenção Primária à Saúde (APS) corresponde a porta de entrada do acesso da população brasileira ao Sistema Único de Saúde (SUS), objetivando primordialmente a proteção e a promoção da saúde, em esferas individuais e coletivamente, enquadrando um formato de atenção composto por cuidados essenciais de saúde fundamentados em evidências científicas, metodologias e tecnologias práticas que sejam acessíveis ao coletivo, por intermédio do incentivo da colaboração popular. O sistema de APS na saúde brasileira, norteado pela Estratégia Saúde da Família (ESF), promove a atenção integral da população nos âmbitos comunitário, familiar e pessoal, abrangendo tanto a iminência da morte quanto a assistência em seu acontecimento. (SOUZA et al., 2015) (MARCUCCl et al., 2016).

A APS foi forçada a se adaptar a composição atual da estruturação populacional do Brasil. O contexto sanitário e social do desenvolvimento epidemiológico e demográfico brasileiro, marcado por um gradativo envelhecimento da população, além do crescimento do predomínio de patologias crônicas e progressivas, como as doenças cardíacas e oncogênicas, corroborou para os cuidados ao fim da vida, necessitando de uma reestruturação da saúde. Nessas circunstâncias, os cuidados paliativos podem ocorrer no setor de internação hospitalar, de acompanhamento em ambulatório ou a nível domiciliar. (SOUZA et al., 2015) (QUEIROZ et al., 2013).

Os cuidados paliativos (CP) correspondem a uma atuação de cuidado em saúde, promovendo melhores condições de vida através do amparo emocional para o paciente e sua família nas circunstâncias em que há ameaça a vida e imediação a morte, proporcionando a redução do sofrimento. Enfatiza-se que os CP demandam o estabelecimento de uma relação interpessoal entre quem é cuidado e os que cuidam, necessitando de uma equipe multidisciplinar para a oferta de uma assistência harmônica, direcionada ao paciente sem
Keywords: palliativecare, primaryhealth and basichealth.

possibilidades terapêuticas curativas e estendida também a sua família. Neste sentido, são os profissionais da atenção básica quem acolhem emocionalmente a situação do paciente, de seus familiares e da rede de cuidado. Logo, o entendimento desses profissionais, presume-se em olharmos para as múltiplas relações dentro do que é vivenciado por todos os seres envoltos em um fenômeno e não somente por um indivíduo que se encontra envolvido com o fenômeno. (MAQUES; BULGARELLI, 2020).

O desempenho dos médicos de família e comunidade na prática dos Cuidados Paliativos ocorre de forma proveitosa e assertiva, visto que eles presenciam o envelhecimento e a debilitação dos pacientes, o empenho dos familiares para atenderem às novas demandas de cuidado de seus entes, os anseios, a instabilidade econômica e, mediante a isso, não podem se afastar nessa situação: são justamente nessas situações que toda a abrangência do ofício da APS e da Estratégia de Saúde da Família (ESF) se evidencia. Não há outro serviço de saúde que possa acompanhar essas famílias com tanta especialidade e prestar os cuidados da paliação com acolhimento, presença constante e orientação. (SILVA, 2014).

Nesse âmbito, entende-se que os CP demandam a necessidade da prestação de assistência, não obstante, essencialmente em assegurar a dignidade e qualidade de vida dos pacientes enquanto seres humanos. O sistema de atenção à saúde que até este tempo privilegia as ações curativas necessita aderir a um novo formato, em que a enfermidade não apenas é curável, como também deve ser tratável, ao qual transcorre através da ética do cuidado. (RABELLO; RODRIGUES, 2010).

Sendo assim, almeja-se maximizar os conhecimentos para o processo de aprendizagem dos cuidados paliativos na atenção primária a saúde e os fatores intrínsecos a este que norteiam o trabalho prestado por médicos de família e comunidade aos pacientes paliativos do Sistema Único de Saúde. Ademais, é necessária a formação 
de médicos preparados para enfrentar a morte e o percurso até seu acontecimento de forma consciente, ressignificando-os como algo inerente a vivência humana e não como objeto de contradição a sua definição. (COBO et al., 2019).

\section{OBJETIVOS}

Revisão da literatura sobre os cuidados paliativos promovidos por médicos de família e comunidade na atenção primária à saúde brasileira.

\section{MÉTODOS}

Este estudo é uma revisão bibliográfica, que foi realizado entre julho e agosto de 2020 e, para o desdobramento do mesmo, foram feitas buscas bibliográficas nas bases de dados a seguir: Scientific Library Online (Scielo) e Literatura Latino-Americana e do Caribe em Ciências da Saúde (Lilacs).

As buscas foram realizadas utilizando terminologias específicas baseadas no tema. As palavras chaves empregadas foram "cuidados paliativos", "atenção básica" e "atenção primária à saúde".

\section{REVISÃO BIBLIOGRÁFICA IMPLEMENTAÇÃO DOS CUIDADOS PALIATIVOS NA ATENÇÃO BÁSICA}

Embora tenha uma pluralidade nas intervenções sugeridas para o avanço da qualidade de vida na contemporaneidade, permitido pela evolução tecnológica, a autenticidade da vivência em saúde entra em contraste com novas carências na assistência à saúde. Além disso, devido ao aparecimento de novas enfermidades, novas circunstâncias de vida e, consequentemente, novos meios e possibilidades terapêuticas, que é o caso da assistência em cuidados paliativos no âmbito da APS. Essas novas necessidades fazem com que precise do SUS como uma assertiva de apoio e pactuação para integração de ações, até mesmo em cunho intersetorial. (MARCUCCl et al., 2016).

A entrega de uma assistência de qualidade aos pacientes em situação terminal e seus cuidadores, na grande maioria das vezes, é limitada pela existência de uma extensa e plural demanda na atenção primária à saúde. No vínculo do hospital com a ESF, aspecto habitual a profissionais e familiares, predomina o entendimento de que, depois de diagnosticado com uma doença em estágio terminal, os pacientes são encaminhados pelo hospital às suas casas pois não há mais condutas cabíveis, sem comunicar previamente os profissionais designados ao acompanhamento domiciliar. (QUEIROZ et al., 2013).

Um estudo brasileiro que objetivou entender o estado atual do cenário da prática dos cuidados paliativos apontou que os médicos, majoritariamente, ligaram essa área apenas ao alívio do sofrimento físico e qualidade de vida, negligenciando os aspectos, psicológicos, espirituais e sociais, além de não enquadrar a família do enfermo na abordagem de um serviço de cuidados paliativos. Frisa-se que a questão dos CP na APS não se resume a aspectos técnicos, porém estende-se aos obstáculos éticos tais como: a decisão sobre o local em que o paciente irá morrer, a maneira com que os profissionais da equipe de saúde vivenciam a experiência de assistir à morte e ao luto, além da identificação e administração das condições familiares e do paciente. (SOUZA et al., 2015) (RIBEIRO; POLES, 2019).

Os médicos que trabalham nas Unidades Estratégia Saúde da Família demonstraram nos seus relatos as inúmeras possibilidades de atuação que existe neste cenário quando é pensado na melhora da qualidade de vida e de morte dos pacientes que se encontram em $\mathrm{CP}$, apesar do pequeno quantitativo de pacientes. É ressaltado pela literatura as várias carências dos pacientes em final de vida, as quais abrangem receber cuidados para o controle da dispnéia, constipação intestinal e náuseas, dor, por exemplo, além de receber amparo emocional, espiritual e social, acessar a informações, participar das discussões sobre a sua vida e condição de saúde e buscar o significado da vida. Ademais, o papel da ESF relaciona-se aos procedimentos de retaguarda, como articular, junto à rede de serviços, as internações e outros procedimentos necessários a qualidade de vida do paciente; em fornecer deslocamento para o doente possibilitando o acesso a serviços mais complexos; em orientar e amparar a família; em identificar as necessidades, abrir mão de medicamentos e outros subterfúgios, como sonda e colchão d'água; e, quando em caso de falecimento fornecer o atestado de óbito. (QUEIROZ et al., 2013) (RIBEIRO; POLES, 2019).

\section{DIFICULDADES DA IMPLEMENTAÇÃO DOS CUIDADOS PALIATIVOS NA ATENÇÃO BÁSICA}

As principais dificuldades na assistência 
domiciliar consistem em estabelecer uma relação com a família, aprender a trabalhar em equipe junto à comunidade, estabelecer uma rede de apoio social e ter retaguarda efetiva com os demais serviços de apoio aos pacientes (QUEIROZ et al., 2013).

Sabe-se que um dos obstáculos encontrados é em relação à oferta de serviços de $\mathrm{CP}$, que mesmo com a promulgação da legislação específica, a qual estabelece, no SUS, o CP e o atendimento/internação domiciliar, ainda é um serviço pouco ofertado. (SILVA, 2014).

A APS, assim como o CP, tem como objetivo o cuidado integral, tanto do paciente como de sua família, orientando e ajudando os mesmos como também tratando e cuidando deles para que possuam uma qualidade de vida melhor nesta situação. No entanto, sem o treinamento adequado das equipes de $C P$, da presença das mesmas no SUS, é difícil identificar os pacientes que necessitam de $\mathrm{CP}$, por conseguinte, é difícil a sua implementação na atenção básica e a realização de um treinamento com esses profissionais (MARCUCCl et al., 2016).

Além disso, existe a falta de informação sobre a quantidade de pacientes que necessitam de $C P$ na ESF, como também informações das suas condições sociais, das principais doenças que acometem o paciente, do grau de dependência do mesmo no dia a dia, entre muitas outras situações as quais precisam ser explicitadas no prontuário (MARCUCCl etal., 2016).

Acrescenta-se a essas situações o âmbito familiar, o qual nem sempre está aberto aos CP. Dessa forma, é importante que o profissional da ESF tenha compreensão e faça seu trabalho, ajudando tanto o paciente como a família (QUEIROZ et al., 2013).

\section{AÇÕES PARA AJUDAR NA IMPLEMENTAÇÃO}

A APS é capaz de organizar, de forma que ajude no prosseguimento do paciente em CP na sua casa, evitando, assim, que ocorra o distanciamento da família em momento delicados, como é o final da vida (SILVA, 2014).

Os atendimentos domiciliares aos pacientes em CP necessitam de uma disponibilidade de tempo, para os mesmos terem uma atenção devida, permitindo, portanto, um vínculo e maior esclarecimento sobre a situação do paciente para o mesmo, quando possível, e para a família. Sendo assim, é imprescindível uma agenda que favoreça a disponibilidade para este atendimento (OLIVEIRA et al., 2019).

O profissional de saúde precisa entender a dinâmica da família do enfermo e o papel que ele desempenha. A família, quando bem abordada e trabalhada, pode ser vista como um agente terapêutico eficaz, promovendo elo entre o paciente e a equipe de saúde, mas, caso isso não ocorra, pode ter influência negativa na evolução do quadro do doente. Dessa forma, o agente de saúde deve auxiliar a família nas decisões a serem resolvidas em diversas circunstâncias (QUEIROZ et al., 2013).

Ao decidir que o paciente ficará em seu domicílio, é necessário que a ESF dê a assistência necessária, acompanhe a organização e analise as dificuldades enfrentadas pelos familiares. É preciso lembrar que um dos objetivos da ESF é o acompanhamento longitudinal. Sendo assim, essa abordagem é favorecida, mas é essencial que se avalie e que isso resulte em ações de apoio da equipe (QUEIROZ et al., 2013).

\section{CONCLUSÃO}

Visto que a APS tem como objetivo a promoção e a proteção da saúde, tanto individual quanto coletivamente, por meio de cuidados essenciais, é fundamental a aplicação de cuidados paliativos aos pacientes, que se encontram em contexto de risco de vida e proximidade com a morte, e seus respectivos familiares, uma vez que tais cuidados são empregados a fim de melhorar as condições de vida, aliviar os sofrimentos e ocasionar conforto emocional aos pacientes. Para que isso ocorra, é necessário o reconhecimento da importância dessa prática e do comprometimento do acompanhamento, além da preparação e treinamento adequado dos profissionais e das equipes de CP na APS, que realizam trabalho multidisciplinar, com a finalidade de melhorar a saúde e o bem estar do paciente.

Após analisar os obstáculos que precisam ser vencidos para a aplicação dos $\mathrm{CP}$, é de essencial o estabelecimento de relação entre o profissional e a família do paciente, ou seja, a atuação em conjunto das equipes junto à família, dado que esse vínculo, quando bem realizado, pode ser benéfico para a eficácia dos cuidados paliativos, que estão relacionados a todos os aspectos que envolvem o paciente, desde o sofrimento físico a aspectos sociais, familiares, emocionais, psicológicos e 
espirituais. Além disso, para que os pacientes recebam a melhor assistência possível, os profissionais precisam oferecer disponibilidade de tempo para o atendimento, favorecendo a criação de vínculos e possibilitando esclarecimentos sobre a situação do paciente, tanto para ele mesmo quanto para a família.

\section{REFERÊNCIAS}

COBO, Viviane de Almeida et al. Cuidados Paliativos na Atenção Primária à Saúde: perspectiva dos profissionais de saúde. Bol. - Acad. Paul. Psicol., São Paulo, v. 39, n. 97, p. 225-235, dez. 2019. Disponível em <http://pepsic.bvsalud.org/scielo.php?script=sci_arttext\&pid=S1415-711X2019000200008\&lng=pt\&nrm=iso>. acesso em 10 ago. 2020.

MARCUCCI, Fernando Cesar Iwamoto et al. Identificação de pacientes com indicação de Cuidados Paliativos na Estratégia Saúde da Família: estudo exploratório. Cad. saúde colet., Rio de Janeiro, v. 24, n. 2, p. 145-152, June 2016. Disponível em:<http://www.scielo.br/scielo.php?script=sci_arttext\&pid=S1414-

462X2016000200145\&Ing=en\&nrm=iso>. Acesso em: 10 Aug. 2020. https://doi.org/10.1590/1414462X201600020012.

MARQUES, Fernanda Pasquetti; BULGARELLI, Alexandre Fávero. Os sentidos da atenção domiciliar no cuidado ao idoso na finitude: a perspectiva humana do profissional do SUS. Ciênc. saúde coletiva, Rio de Janeiro, v. 25, n. 6, p. 2063-2072, June 2020. Disponível em:<http://www.scielo.br/scielo.php?script=sci_arttext\&pid=S141381232020000602063\&Ing=en\&nrm=iso>. Acesso em: 10 Aug. 2020. EpubJune 03, 2020.

http://dx.doi.org/10.1590/1413-81232020256.21782018.

OLIVEIRA, Talita de; BOMBARDA, Tatiana Barbieri; MORIGUCHI, Cristiane Shinohara. Fisioterapia em cuidados paliativos no contexto da atenção primária à saúde: ensaio teórico. Cad. saúde colet., Rio de Janeiro, v. 27, n. 4, p. 427-431, Dec. 2019. Disponível em:<http://www.scielo.br/scielo.php?script=sci_arttext\&pid=S1414462X2019000400427\&lng=en\&nrm=iso>. Acesso em: 10 Aug. 2020. EpubNov 14, 2019. https://doi.org/10.1590/1414-462×201900040166.

QUEIROZ, Ana Helena Araújo Bomfim et al. Percepção de familiares e profissionais de saúde sobre os cuidados no final da vida no âmbito da atenção primária à saúde. Ciênc. saúde coletiva, Rio de Janeiro, v. 18, n. 9, p. 2615-2623, Sept. 2013. Disponível em:<http://www.scielo.br/scielo.php?script=sci_arttext\&pid=S141381232013000900016\&lng=en\&nrm=iso>. Acesso em 10 Aug. 2020. https://doi.org/10.1590/S141381232013000900016.

RABELLO, Cláudia Azevedo Ferreira Guimarães; RODRIGUES, Paulo Henrique de Almeida. Saúde da família e cuidados paliativos infantis: ouvindo os familiares de crianças dependentes de tecnologia. Ciênc. saúde coletiva, Rio de Janeiro, v. 15, n. 2, p. 379-388, Mar. 2010. . Disponível em:<http://www.scielo.br/scielo.php?script=sci_arttext\&pid=S1413-81232010000200013\&lng=en\&nrm=iso>. Acesso em 10 Aug. 2020. https://doi.org/10.1590/S1413-81232010000200013.

RIBEIRO, Júlia Rezende; POLES, Kátia. Cuidados Paliativos: Prática dos Médicos da Estratégia Saúde da Família. Rev. bras. educ. med., Brasília, v. 43, n. 3, p. 62-72, July 2019. Disponível em: <http://www.scielo.br/scielo.php?script=sci_arttext\&pid=S0100-55022019000300062\&lng=en\&nrm=iso>. Acesso em: 10 Aug. 2020. Epub May 23, 2019. http://dx.doi.org/10.1590/1981-52712015v43n3rb20180172. SILVA, Mariana Lobato dos Santos Ribeiro. O papel do profissional da Atenção Primária à Saúde em cuidados paliativos. Revista Brasileira de Medicina de Família e Comunidade, Rio de Janeiro, v. 30, n. 9, p. 45-53, jan. 2014. Disponível em: https://www.rbmfc.org.br/rbmfc/issue/view/39/7. Acesso em: 10 ago. 2020.

SOUZA, HiedaLudugério de et al. Cuidados paliativos na atenção primária à saúde: considerações éticas. Rev. Bioét., Brasília, v. 23, n. 2, p. 349-359, Aug. 2015. Disponível em: <http://www.scielo.br/scielo.php?script=sci_arttext\&pid=S1983-80422015000200349\&lng=en\&nrm=iso>. Acesso em: 10 Aug. 2020. https://doi.org/10.1590/1983-80422015232074. 